\title{
Sulle varie forme che possono darsi alle relazioni fra i determinanti di una ma- trice rettangolare.
}

\author{
(Di Ernesto Pascal, a Pavia.)
}

\begin{abstract}
L e relazioni fra $\mathrm{i}$ determinanti di una matrice sono state presentate da vari Autori sotto varie forme diverse. Cosi il VAHLEN ne ha fatto l'argomento di un recente lavoro (Ueber die Relationen zwischen den Determinanten einer. Matrix. Crelle, v. 112 , pag. 306 [1893]); il Nexto ha presentato ultimamente una formola notevole (Zwei Determinantensëtze, Acta Math., v. 17, pag. 199 [1893]), e l'Hunyady ha costruito per scopo geometrico una lunga serie di identità relative a questo soggetto (Ueber einige Determinanten-Gleichungen. Crelle, v. 94, pag. 171 [1882]).

Ora tali relazioni possono riassumersi tutte in una formola unica di tipo semplice, e che non è altro che una delle notissime identità che occorrono nella teoria del calcolo simbolico delle forme algebriche di specie $m$; è formata con una somma algebrica di prodotti di due determinanti di ordine $m$. Qualunque altra relazione fra determinanti dello stesso ordine, o fra determinanti anche di ordine diverso (particolarizzando alcune delle colonne della matrice, col porre eguali a zero tutti gli elementi, meno uno, che si pone invece eguale ad 1, si hanno relazioni fra determinanti di ordine diverso), non può che essere una conseguenza di identità del tipo indicato. Ciò non è che un caso particolare di un teorema più generale che può dimostrarsi per le combinazioni di tipo invariantivo. (V. per es. la mia Memoria: Sopra le relazioni che possono sussistere identicamente fra formazioni simboliche di tipo invariantivo nella teoria generale delle forme algebriche. Memorie dei Lincei, serie $4 . .^{\mathrm{a}}$, v. V, pag. 374 [1888].)

Sebbene però tutte le altre identitì non siano che trasformazioni di quelle indicate, ciò non toglie che queste possono raggrupparsi in maniera da dar luogo a delle formole e dei teoremi notevoli da per sè stessi.
\end{abstract}


Così tutte le note e molteplici formole sui determinanti ad elementi determinanti, sebbene si possano far subito rientrare nella categoria di formole cui si accenna, pure possono poi ancora meglio considerarsi e studiarsi separatamente da quelle. Volendo generalizzare le indicate formole sui determinanti ad elementi determinanti, in modo da farle subito rientrare in quella categoria di formole, occorre studiare dei determinanti formati colle colonne di due determinanti dati, il che cominciò a fare il sig. Proquet alla fine di un lavoro che ha notevoli pregi di fattura. (Analyse combinatoire des déterminants. Journal de ]'Ecole Polyt. V. XXVIII p. 201 [1878].)

Lo scopo di questo lavoro è di presentare alcune altre formole e teoremi nuovi che son venuto trovando, rifacendo per conto mio, questi studi, e che possono stare allato a quelli trovati dagli autori sunnominati e da altri.

\section{§ 1. Cenno sui risultati di VAHLEN. Identità fondamentale.}

Il Vahlev nella citata Nota trova che le relazioni esistenti fra i determinanti di una matrice di $n$ colonne e $m$ linee sono in numero di:

$$
\left(\begin{array}{c}
n \\
m
\end{array}\right)-1-m(n-m)
$$

fra loro indipendenti. Tali relazioni sono espresse nella seguente maniera. Indichiamo in generale con $\left(i_{1}, i_{2}, \ldots i_{m}\right)$ il determinante formato colle colonne $i_{1}, i_{2}, \ldots i_{m}$. Allora si ha:

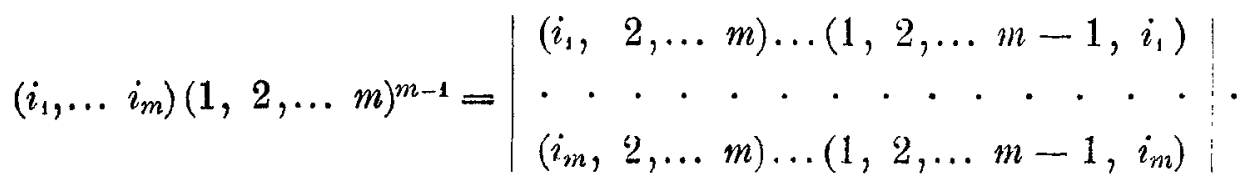

È facile vedere che se due soli degli indici $i$, sono diversi dagli indici $1,2, \ldots m$ allora al secondo membro si ha per fattore $(1,2, \ldots m)^{m-2}$, e quindi, sopprimendo questo fattore si ha una relazione di $2 .^{\circ}$ grado; di tali se ne hanno $\left(\begin{array}{c}n-m \\ 2\end{array}\right)\left(\begin{array}{l}n \\ 2\end{array}\right)$. Similmente se tre soli degli indici $i$ sono diversi da $1,2, \ldots m$, allora si hanno $\left(\begin{array}{c}n-m \\ 3\end{array}\right)\left(\begin{array}{l}n \\ 3\end{array}\right)$ relazioni di $3 .^{\circ}$ grado, e così di seguito.

Formiamo un determinante nella seguente maniera. 
Costruiamo la matrice che ha $m+1$ colonne della matriee data e propriamente quelle di ordini:

$$
i_{1} i_{2} \ldots i_{m} i_{m+1} .
$$

Poniamo poi come ultima linea di questa matrice parziale una linea formata cogli elementi:

$$
\begin{aligned}
& \left(i_{1} \quad j_{1} j_{2} \ldots j_{m-1}\right) \\
& \left(\begin{array}{ll}
i_{2} & j_{1} j_{2} \ldots j_{m-1}
\end{array}\right) \\
& \left(i_{m+1} j_{1} j_{2} \ldots j_{m-1}\right)
\end{aligned}
$$

dove $j_{1} j_{2} \ldots j_{m-1}$ rappresentano gli indici di altre colonne della matrice data (in particolare alcune delle $j$ potrebbero essere eguali alle $i$ ). Si viene così a formare un determinante di ordine $m+1$ il quale è identicamente zero, perchè gli elementi dell'ultima linea sono le medesime combinazioni lineari degli elementi delle linee parallele. Sviluppando questo determinante secondo gli elementi dell'ultima linea si ha:

$$
\mathbf{\Sigma} \pm\left(i_{1} i_{2} \ldots i_{m}\right)\left(i_{m+1} j_{1} j_{2} \ldots j_{m-1}\right)=0,
$$

dove il sommatorio si estende a tutte le permutazioni circolari degli indici $i$, e i segni dei termini sono alternati se $m$ è dispari, e sono tutti positivi se $m$ è pari. È questa la relazione fondamentale dalla quale tutte le altre possono ricavarsi.

Cominciamo col mostrare come le relazioni di $\mathrm{V}_{\triangle B \text { LLEN }}$ avanti eitate si possono ricavare dalle relazioni (A) le quali hanno il vantaggio di essere tutte di 2.0 grado nei determinanti:

Per intenderci più facilmente chiameremo nella relazione (A) elementi circolanti gli indici $i_{1} i_{2} \ldots i_{m-1}$, e elementi fissi gli indici $j_{1} j_{2} \ldots j_{m-1}$. Se tutti gli elementi fissi sono diversi dai circolanti, allora la (A) conterrà $m+1$ termini; se $r$ elementi fissi sono eguali ad altrettanti circolanti, allora $r$ termini si annullano, e restano solo $m-r+1$ termini. Nella (A) poniamo in particolare:

$$
\begin{array}{lll}
i_{1}=1, & i_{2}=2, \ldots & i_{m}=m \\
j_{2}=i_{1}=1, & j_{3}=i_{2}=2, \ldots & j_{m-1}=i_{m-2}=m-2 \\
i_{m+1}=i, & j_{1}=j . &
\end{array}
$$


Allora si ha una relazione a tre termini solo, cioè:

$$
\begin{gathered}
(1,2, \ldots m-2, m-1, m)(i, j, 1, \ldots m-2)+ \\
+(1,2, \ldots m-2, m, i)(m-1, j, 1, \ldots m-2)+ \\
+(1,2, \ldots m-2, i, m-1)(m, j, 1, \ldots m-2)=0 .
\end{gathered}
$$

Questa non è altro che una delle relazioni di $2 .^{\circ}$ grado della formola generale di VAHLEN; quella che si ottiene ponendo in essa $i_{1}=1, \ldots i_{m-2}=m-2$, $i_{m-1}=i, i_{m}=j$.

Passiamo alle relazioni di $3 .^{\circ}$ grado di Vahles. Una di esse è per es.:

$$
\left(1,2, \ldots m-3, i_{m-2} i_{m-1} i_{m}\right)(1,2, \ldots m)^{2}=
$$

$$
=\left|\begin{array}{l}
\left(1,2, \ldots m-3, i_{n-2}, m-1, m\right),\left(1,2, \ldots m-2, i_{n-2}, m\right),\left(1,2, \ldots m-1, i_{m-2}\right) \\
\left(1,2, \ldots m-3, i_{m-1}, m-1, m\right),\left(1,2, \ldots m-2, i_{m-1}, m\right),\left(1,2, \ldots m-1, i_{m-1}\right) \\
\left(1,2, \ldots m-3, i_{m}, m-1, m\right),\left(1,2, \ldots m-2, i_{m}, m\right),\left(1,2, \ldots m-1, i_{m}\right) .
\end{array}\right|
$$

Sviluppiamo questo determinante secondo gli elementi dell'ultima colonna, e teniamo conto delle relazioni simili all'ultima scritta. I minori di $2 .{ }^{\circ}$ ordine compresi nelle due prime colonne di questo determinante sono rispettivamente eguali a:

$$
\begin{aligned}
& +\left(1,2, \ldots m-3, i_{m-2}, i_{m-1}, m\right)(1,2, \ldots m), \\
& -\left(1,2, \ldots m-3, i_{m-2}, i_{m}, \quad m\right)(1,2, \ldots m), \\
& +\left(1,2, \ldots m-3, i_{m-1}, i_{m}, \quad m\right)(1,2, \ldots m),
\end{aligned}
$$

e quindi, sopprimendo il fattore comune $(1,2, \ldots$ m), resta la relazione:

$$
\begin{aligned}
& \left(1,2, \ldots m-3, i_{m-2}, i_{m-1}, i_{m}\right)(1,2, \ldots m-2, m-1, m)- \\
- & \left(1,2, \ldots m-3, i_{m-2}, i_{m-1}, m\right)\left(1,2, \ldots m-2, m-1, i_{m}\right)+ \\
+ & \left(1,2, \ldots m-3, i_{m-2}, i_{m}, m\right)\left(1,2, \ldots m-2, m-1, i_{m-1}\right)- \\
- & \left(1,2, \ldots m-3, i_{m-1}, i_{m}, m\right)\left(1,2, \ldots m-2, m-1, i_{m-2}\right)=0,
\end{aligned}
$$

che si ricara da (A) ponendo:

$$
j_{1}=1, \ldots \quad j_{m-1}=m-1
$$

e

$$
i_{1}=1, \ldots \quad i_{m-3}=m-3, \quad i_{m+1}=m .
$$


Così si potrebbe dimostrare che lo stesso accade per le relazioni di quarto grado, e di grado superiore, e, in generale tutte le possibili relazioni fra $i$ determinanti di una matrice non sono che trasformazioni opportune della formola A.

\section{§ 2. Su di una formola di Netro e su di un'altra ad essa affine (*).}

Consideriamo $m+2$ colonne della matrice data. Indichiamo con $\Delta_{\alpha \beta}$ il determinante che si ottiene sopprimendo le colonne $\alpha, \beta$; allora si ha la relazione identica:

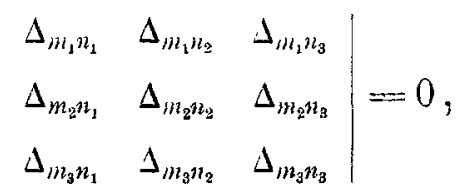

dove $m_{1}, n$, ecc., sono indici scelti fra quelli che compongono la matrice parziale di $m+2$ colonne. Questa è una formola che il sig. Netro ha fatto conoscere in un recente lavoro negli Acta Mathematica (v. XVII, pag. 199).

Ora questa formola discende subito dalla relazione fondamentale (A), e ne discende poi anche un'altra furmola che ha perfetta analogia con questa, ma che nella sostanza è affatto diversa.

Per fissare le idee supponiamo che le $m-4$ colonne che si ottengono quando dalle $m+2$ scelte si tolgono quelle di indiei $m_{3} m_{2} m_{3} n_{1} n_{2} n_{3}$ sieno $i_{1} i_{2} \ldots i_{m-4}$.

Come caso particolare della (A) si costruisca la relazione a tre termini:

$$
\begin{aligned}
& \left(i_{1} \ldots i_{m-4} m_{2} m_{3} n_{2} n_{3}\right)\left(i_{1} \ldots i_{m-4} m_{1} m_{2} m_{3} n_{3}\right)+ \\
+ & \left(i_{1} \ldots i_{m-4} m_{2} m_{3} n_{3} n_{1}\right)\left(i_{1} \ldots i_{m-4} m_{1} m_{3} m_{3} n_{2}\right)+ \\
+ & \left(i_{1} \ldots i_{m-4} m_{2} m_{3} n_{1} n_{2}\right)\left(i_{1} \ldots i_{m-4} m_{1} m_{2} m_{3} n_{3}\right)=0
\end{aligned}
$$

cioè colla notazione ora introdotta:

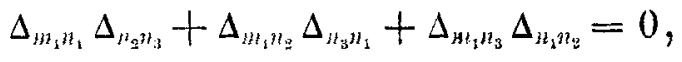

e mutando $m_{1}$, in $m_{2}$ e in $m_{3}$, e osservando che i secondi fattori di ciascun termine restano inalterati, si riconosce subito che il determinante dei primi fattori deve essere zero.

(*) Da non confondor'si con quella che forma l'argomento di una mia Nota ultimamente stampata ai Lincei (1896), la quale porta lo stesso titolo di questo paragrafo. 
Invece in questa stessa formola si cambi $m_{1}$ in $m_{1}^{\prime}$ e poi ancora in $m^{\prime \prime}{ }_{1}$, dove $m^{\prime}{ }_{1} m^{\prime \prime}{ }_{1}$ sono indici nuovi scelti fra quelli che appartengono alle altre colonne non ancora considerate. Si consideri una matrice con $m+4$ colonne:

$$
i_{1} \ldots i_{m-4} m_{1} m_{2} m_{3} n_{1} n_{2} n_{3} m_{1}^{\prime} m^{\prime \prime}{ }_{1} \text {. }
$$

Poniamo $m_{2}=i_{m \cdot 3} m_{3}=i_{m-2} ; i$ determinanti che entrano allora nelle tre formole hanno tutti per parte comune le $m-2$ colonne $i_{1} \ldots i_{m-2}$. Indichiamo con $\Delta_{\alpha \beta}^{\prime}$ un determinante formato aggiungendo a queste $m-2$ colonne fisse, le due colonne $\alpha, \beta$. La prima delle relazioni di sopra prende la forma:

$$
\Delta_{n_{2} n_{3}}^{\prime} \cdot \Delta_{m_{1} n_{1}}^{\prime}+\Delta_{n_{3} n_{1}}^{\prime} \cdot \Delta_{m_{1} n_{2}}^{\prime}+\Delta_{n_{1} n_{2}}^{\prime} \cdot \Delta_{m_{1} n_{\mathrm{t}}}^{\prime}=0,
$$

e le altre due si ottengono mutando $m_{1}$ in $m_{1}^{\prime}, m^{\prime \prime}{ }_{1}$.

Si ha quindi:

$$
\begin{aligned}
& \Delta_{m_{1} n_{1}}^{\prime} \quad \Delta_{m_{1} n_{2}}^{\prime} \quad \Delta_{m_{1} n_{3}}^{\prime} \\
& \Delta_{m_{1}^{\prime} n_{1}}^{\prime} \quad \Delta_{m_{1}^{\prime} n_{1}}^{\prime} \quad \Delta_{m_{1}^{\prime} n_{3}}^{\prime}=0 \text {, } \\
& \Delta_{n^{\prime \prime} n_{1}}^{\prime} \quad \Delta_{m n_{1}^{\prime \prime} n_{3}} \quad \Delta_{m_{1}^{\prime \prime} n_{3}}^{\prime}
\end{aligned}
$$

che formalmente costituisce una relazione analoga a quella di Nsтto, ma che nella sostanza ne è diversa, perchè mentre nella relazione di Nerro gli elementi sono determinanti ottenuti sopprimendo due colonne in una matrice di $m+2$ colonne, in questa relazione gli elementi sono determinanti ottenuti aggiungendo due colonne ad una matrice di $m-2$ colonne.

\section{\$ 3. Relazioni fra i determinanti formati colle colonne di due determi- nanti dati. Continuazione delle ricerche di PICQUET. Estensione di un teorema di Sycvester.}

Sieno dati due determinanti di ordine $m$ :

$$
\begin{aligned}
& A=\left(a_{1} a_{2} \ldots a_{m}\right) \\
& B=\left(b_{1} b_{2} \ldots b_{m}\right) .
\end{aligned}
$$

Si può formare una matrice di $2 m$ colonne e $m$ linee ponendo le due matrici dei due determinanti l'una accanto all'altra. 
Scegliamo in tutti i modi possibili $k$ colonne di $A$ e al loro posto sostituiamo $l e$ colonne di $B$ scelte in tutti i modi possibili; abbiamo in tutto $\left(\begin{array}{l}m \\ k\end{array}\right)^{2}$ determinunti formati con $m-k$ colonne di $A$ e $k$ di $B$; questi possono disporsi in una matrice quadrata e formare a loro volta un determinante che chiameremo $D_{n-k}$; analogamente poi può formarsi il determinante $D_{k}$ i cui elementi sono determinanti contenenti $k$ colonne di $A$ e $n-k$ di $B$; questi due determinanti sono evidentemente degli stessi ordini. Se $B$ diventa:

$$
\left|\begin{array}{cccc}
1 & 0 & 0 & \ldots \\
0 & 1 & 0 & \ldots \\
0 & 0 & 1 & \ldots \\
\ldots & \ldots
\end{array}\right|,
$$

allora in particolare $D_{m-k}$ e $D_{k}$ diventano i determinanti formati coi minori di ordine $m-k$ e di ordine $k$ del deterninante $A$.

Su questi determinanti $D$, il Picqoen dimostra i due teoremi:

1. Sia $D_{k}$ che $D_{m-k}$ sono ciascuno eguali al prodotto di una potenza di $A$ per una potenza di $B$ :

$$
D_{k}=A^{\left(\begin{array}{c}
m-1 \\
k-1
\end{array}\right)} B^{\left(\begin{array}{c}
m-1 \\
k
\end{array}\right)} \text {. }
$$

2. Ogni minore di ordine $r$ di $D_{k}$ è eguale al prodotto del complemento del suo omologo in $D_{m-k}$ per una potenza di $A$ e una potenza di $B$; propriamente per:

$$
A^{r-\left(\begin{array}{c}
m-1 \\
k
\end{array}\right)} B^{r-\left(\begin{array}{c}
m-1 \\
k-1
\end{array}\right)} \text {. }
$$

(Si suppongono naturalmente ordinati convenientemente gli elementi di $D_{k}$ e $D_{m-k}$.)

A questi teoremi di Proquet noi ne aggiungiamo alcuni altri.

Consideriamo $D_{m-1}$ che è di ordine $m$. Noi dimostreremo che:

3. Ogni minore di ordine l: di $D_{m-1}$ è eguale a $A^{k-1}$ moltiplicato per un elemento del determinante $D_{m-k}$.

Questo teorema non è altro che la estensione di quello sugli ordinari determinanti reciproci.

In effetti un minore di $2 .^{\circ}$ ordine di $D_{m l^{-1}}$ è (salvo i nomi degli elementi) riducibile sempre al tipo:

$$
\left|\begin{array}{c}
\left(a_{1} \ldots a_{m-2} a_{m-1} b_{1}\right),\left(a_{1} \ldots a_{m-2} a_{m} b_{1}\right) \\
\left(a_{1} \ldots a_{m-2} a_{m-1} b_{2}\right),\left(a_{1} \ldots a_{m-2} a_{m} b_{2}\right)
\end{array}\right|
$$


che per effetto della identità fondamentale può trasformarsi in:

$$
\left(a_{1} \ldots a_{m-2} a_{m-1} a_{m}\right)\left(a_{1} \ldots a_{m-2} b_{1} b_{2}\right)
$$

di cui il primo fattore è $A$ e l'altro è un elemento di $D_{m \cdots ?}$. Consideriamo ora un minore di $3 .^{\circ}$ ordine di $D_{m-1}$. Salvo i nomi degli elementi, esso è sempre del tipo:

$$
\begin{aligned}
& \left(a_{1} \ldots a_{m-3} a_{m-2} a_{m-1} b_{1}\right),\left(a_{1} \ldots a_{n-3} a_{m-1} a_{m} b_{1}\right),\left(a_{1} \ldots a_{m-3} a_{m} a_{m-2} b_{1}\right) \\
& \left(a_{1} \ldots a_{n-3} a_{m-2} a_{m-1} b_{2}\right),\left(a_{1} \ldots a_{m-3} a_{m-1} a_{m} b_{2}\right),\left(a_{1} \ldots a_{m-3} a_{m} a_{m-2} b_{2}\right) \\
& \left(a_{1} \ldots a_{m-3} a_{m-2} a_{m-1} b_{3}\right),\left(a_{1} \ldots a_{m-3} a_{m-1} a_{m} b_{3}\right),\left(a_{1} \ldots a_{m-3} a_{m} a_{m-2} b_{3}\right)
\end{aligned}
$$

Sviluppandolo secondo gli elementi dell'ultima linea, e osservando che ai minori di $2 .^{\circ}$ ordine compresi nelle due prime linee, si può applicare il risultato della dimostrazione già fatta, si ha:

$$
\begin{aligned}
& A\left[\left(a_{1} \ldots a_{m-3} a_{m-2} a_{m-1} b_{3}\right)\left(a_{1} \ldots a_{m-3} a_{m} \quad b_{1} b_{2}\right)-\right. \\
& -\left(a_{1} \ldots a_{m-3} a_{m-1} a_{m} \quad b_{3}\right)\left(a_{1} \ldots a_{m-3} a_{m-2} b_{1} b_{2}\right)+ \\
& \left.+\left(a_{1} \ldots a_{m-3} a_{m} \quad a_{m-2} b_{3}\right)\left(a_{1} \ldots a_{m-3} a_{m-1} b_{1} b_{2}\right)\right],
\end{aligned}
$$

che, per effetto della solita identità fondamentale, è uguale a:

$$
A^{2}\left(a_{1} \ldots \alpha_{m-3} b_{1} b_{2} b_{3}\right)
$$

cioè $A^{2}$ moltiplicato per un elemento di $D_{m-3}$. Si vede che questo procedimento può continuarsi e darà sempre l'analogo risultato. Il teorema proposto resta così dimostrato in generale.

Nella teoria dei determinanti formati coi minori di un altro è noto il seguente teorema di Symvester. (V. per es. D'Ovidio, Acc. 'Torino 1876-77-90; PICQUeT, op. cit.)

Indichiamo con $\Delta_{m-k}$ il determinante formato coi minori di ordine $m-k$ di un determinante dato $\Delta$ di ordine $m$. Formiamo quel minore $\Delta_{i k k}$ di $\Delta_{m-k}$, i cui elementi sieno minori contenenti sempre $m-\lambda$ linee e colonne fisse di $\Delta$, e $\lambda-k$ linee e colonne rariabili $(\lambda>h)$. Il minore $\Delta_{i k} \dot{e}$ di ordine $\left(\begin{array}{l}\lambda \\ k\end{array}\right)$ ed esso è eguale ad una potenza di $\Delta$ moltiplicata per una potenza di quel minore di $\Delta$ racchiuso dalle $m-\lambda$ linee e colonne fisse.

Ora vediamo in che modo potrà estendersi questo teorema. 
Consideriamo $D_{m-k}$ e un suo minore formato nel seguente modo: i suoi elementi contengano tutti $m-\lambda$ colonne fisse di $A$ e $\lambda-k$ colonne variabili $(\lambda>k)$, e poi $k$ colonne di $B$ scelte fra $\lambda$ assegnate.

Un tal minore lo chiameremo $D_{\lambda, k}$ ed è di ordine $\left(\begin{array}{l}\lambda \\ k\end{array}\right)$.

Moltiplichiamo ogni suo elemento per $A^{k-1}$; tutto il minore resterd moltiplicato per:

$$
A^{\left(\begin{array}{l}
\lambda \\
k
\end{array}\right)(k-1)}
$$

In forza del teorema ora dimostrato, ogni elemento suo diventa un certo minore di ordine $k$ di $D_{n-1}$, e propriamente un minore i cui elementi sono determinanti contenenti tutte le $m-\lambda$ colonne fisse di $A$, e non contenenti mai le $m-\lambda$ colonne escluse di $B$.

Ora in $D_{m-1}$ tutti gli elementi cosi formati, costituiscono un minore di ordine $\lambda$ che chiameremo $D^{(2)}$; abbiamo dunque che, dopo la moltiplicazione indicata, gli elementi di $D_{\lambda k}$ diventano minori di ordine $k$ di $D^{(\lambda)}$, e si ha quindi il determinante formato coi minori di ordine $k$ di $D^{(\lambda)}$, il quale, come si sa, è eguale ad una potenza di $D^{(2)}$.

Abbiamo dunque la formola:

$$
A^{\left(\begin{array}{l}
\lambda \\
k
\end{array}\right)(k-1)} D_{\lambda k}=\left[D^{(\lambda)}\right]^{\left(\begin{array}{c}
\lambda-1 \\
k-1
\end{array}\right)}
$$

Intanto a $D^{(\lambda)}$, come minore di $D_{n-1}$, puঠ applicarsi ancora il teorema sopra dimostrato, e si ha che $D^{(\lambda)}$ è eguale a $A^{\lambda-1}$ moltiplicato per quell'elemento di $D_{m-\lambda}$ che contiene tutte le $m-\lambda$ colonne fisse di $A$ e le $\lambda$ scelte di $B$. Supposto che le $m-\lambda$ colonne fisse di $A$ sieno indicate con $a_{2} a_{2} \ldots a_{m-\lambda}$, e sieno indicate con $b_{m-\lambda+1} \ldots b_{m}$ le colonne scelte di $B$, tale elemento resta rappresentato con:

$$
\left(a_{1} \ldots a_{m-\lambda} b_{m-\lambda+1} \ldots b_{m}\right)
$$

Raccogliendo si ha dunque infine la formola:

$$
D_{\lambda k}=A^{\left(\begin{array}{c}
\lambda-1 \\
b_{k}
\end{array}\right)} \cdot\left(a_{1} \ldots a_{m-\lambda} b_{m-\lambda+1} \ldots b_{m}\right)^{\left(\begin{array}{l}
\lambda-1 \\
k-1
\end{array}\right)}
$$

che è una formola analoga a quella del citato teorema di Syltestre, il quale se ne ricava come caso particolare se il determinante $B$ si suppone Annali di Matematica, tomo XXIV. 
eguale a:

$$
\left.\mid \begin{array}{llll}
1 & 0 & 0 & \ldots \\
0 & 1 & 0 & \ldots \\
0 & 0 & 1 & \ldots \\
\ldots & \ldots
\end{array}\right]
$$

Possiamo dunque conchiudere:

4. Separiamo le colonne di $A$ e $B$ in due categorie, $m-\lambda$ nell'una $e \lambda$ nell'altra. Formiamo tutti $i$ determinanti colle $m-\lambda$ colonne di $A$, altre $\lambda-k d i A$, e le rimanenti $k d i B$ scelte fra le $\lambda$ della seconda categoria; presi come elementi questi determinanti e opportunamente ordinati si costruisca con essi il determinante di ordine $\left(\begin{array}{l}\lambda \\ k\end{array}\right)$. Questo sarà eguale alla potenza $\left(\begin{array}{c}\lambda-1 \\ k\end{array}\right)^{m a}$ di A per la potenza $\left(\begin{array}{l}\lambda-1 \\ k-1\end{array}\right)^{m a}$ del determinante formato colle $n-\lambda$ colonne di $A$ e colle $\lambda$ di $B$.

Al minore $D_{\lambda k}$ di $D_{m-k}$ si pud far corrispondere in $D_{k}$ il complemento del suo omologo che è di ordine:

$$
\left(\begin{array}{l}
m \\
k
\end{array}\right)-\left(\begin{array}{l}
\lambda \\
k
\end{array}\right)
$$

e, per il teorema citato di PicQuex (teor. 2), questo è eguale a:

$$
D_{\lambda k} \cdot A^{\left(\begin{array}{c}
m \\
k
\end{array}\right)-\left(\begin{array}{l}
\lambda \\
k
\end{array}\right)-\left(\begin{array}{c}
m-1 \\
k
\end{array}\right)} B^{\left(\begin{array}{c}
m \\
k
\end{array}\right)-\left(\begin{array}{l}
\lambda \\
k
\end{array}\right)-\left(\begin{array}{c}
m-1 \\
k-1
\end{array}\right)}
$$

che, in forza del teorema dimostrato, è:

$$
A^{\left(\begin{array}{c}
m \\
k
\end{array}\right)-\left(\begin{array}{l}
\lambda \\
k
\end{array}\right)+\left(\begin{array}{c}
\lambda-1 \\
k
\end{array}\right)-\left(\begin{array}{c}
m-1 \\
k
\end{array}\right)} B{ }^{\left(\begin{array}{c}
m \\
k
\end{array}\right)-\left(\begin{array}{l}
\lambda \\
k
\end{array}\right)-\left(\begin{array}{c}
m-1 \\
k-1
\end{array}\right)}\left(a_{1} \ldots a_{m-\lambda} b_{m-\lambda+1} \ldots b_{m}\right)^{\left(\begin{array}{l}
\lambda-1 \\
k-1
\end{array}\right),}
$$

che è eguale a:

$$
A^{\left(\begin{array}{c}
m-1 \\
k
\end{array}\right)-\left(\begin{array}{l}
\lambda-1 \\
k-1
\end{array}\right)} B^{\left(\begin{array}{c}
m-1 \\
k
\end{array}\right)\left(\begin{array}{l}
\lambda \\
k
\end{array}\right)}\left(a_{1} \ldots a_{m-\lambda} b_{m} \lambda+1 \ldots b_{m}\right)^{\left(\begin{array}{l}
\lambda-1 \\
k-1
\end{array}\right)}
$$

Abbiamo dunque:

5. Formiamo il minore di $D_{k}$ che abbia per elementi, determinanti contenenti $k$ colonne $d i A$ Non sckLte tutre fra le $\lambda$ della seconda categoria, e $m-k$ colonne di $B$ fra le quali non sono mai comprese тuTre le $m-\lambda$ della prima categoria; fra le colonne compaia sempre almeno una colonna di A scelta fra quelle della prima categoria, e non compaia aLMENO ONA 
delle colonne di $B$ fra quelle della prima categoria. Tal minore così formato è di ordine $\left(\begin{array}{l}m \\ k\end{array}\right)-\left(\begin{array}{l}\lambda \\ k\end{array}\right)$ e si esprime colla formola soprascritta.

Questa formola è l' estensione di un teorema del D'Ovidro (loc. cit.).

\section{$\S$ 4. Estensione di un teorema dato dal sig. Nerro come estensione della cosiddetta formola di scomposizione di LAPLACE.}

Il sig. Netro in un lavoro intitolato Erweiterung des Laplaceschen Determinunten-Zerlegungssutzes (Crelle, v. 114, pag. 345), dimostra il seguente teorema:

In un determinante dato di ordine $n$ sopprimiamo $m-\lambda$ linee e colonne, e facciamo la scomposizione del determinante restante secondo la cosiddetta regola di LAPIACE, cioè facciamo la somma dei prodotti con segni opportuni di tutti $i$ minori compresi in $k_{1}$ linee, per tutti quelli compresi in $k_{2}$ linee, e così di seguito, per tutti quelli compresi in $k_{i}$ linee, dove $k_{1}+\cdots+k_{i}=\lambda$, e due fattori di un prodotto non contengono mai in comune una colonna del determinante primitivo. Il risultato di questa somma sarà il determinante delle $\lambda$ colonne e linee non soppresse. Se ora $i$ vari fattori dei vari termini di questa somma si intendono orlati delle $m-\lambda$ linee e colonne soppresse, allora la somma rappresenterà invece il prodotto di tutto il determinante primitivo per la potenza $(i-1)^{m a}$ del minore formato dalle $m-\lambda$. linee e colonne fisse.

Di questo teorema jo ho dato in un altro lavoro $\left({ }^{*}\right)$ una dimostrazione semplice, e ho trovato poi anche un altro teorema che è ad esso affine, e che consiste in questo: si faccia la somma di prodotti come nella regola di $\mathrm{LA}_{\mathrm{A}}$ PLACE; poi ad ogni fattore si sostituisca il suo minore complementare; indi si orlino tutti i fattori colle solite $m-\lambda$ linee e colonne fisse; il risultato della somma sarà allora, viceversa, la potenza $(i-1)^{m a}$ del determinante dato per la prima potenza del minore formato colle $n-\lambda$ linee e colonne fisse.

Estendiamo ora questi teoremi secondo il punto di vista indicato nei paragrafi precedenti.

Separiamo, come avanti, le colonne di due determinanti dati $A, B$ di ordine $m$, in due categorie, $m-\lambda$ nella prima e $\lambda$ nella seconda; per fissare

(*) V. Nota nei Rend. dei Lincei. Marzo, 1896. 
le idee supponiamo che si separino le prime $m-\lambda$ dalle ultime $\lambda$. Le $\lambda$ di $B$ dividiamole in $k_{1}+k_{2}+\cdots+k_{i}=\lambda$. La generalizzazione del teorema è la seguente:

6. Formiamo un determinante colle $m-\lambda$ colonne fisse $d i A$, e poi altre $k_{1}$ di $A$ in tutti $i$ modi possibili, e le $\lambda-k_{1}$ colonne di $B$ già fissate; poi un altro determinante colle $m-\lambda$ colonne fisse $d i A$, con altre $k_{2}$ colonne di $A$ (diverse tutte dalle $k_{1}$ già considerate), e colle altre $\lambda-k_{2}$ colonne di $B_{2}$ fissate, e così proseguiamo per i volte di seguito; poi facciamo la somma algebrica dei prodoti di questi i determinanti tali che in ciascun termine due fattori non abbiano mai in comune altre colonne di $A$ che le $m-\lambda$. Se supponiamo che $B$ diventi cogli elementi zero, meno quelli della diagonale principale che siano eguali ad 1 , allora si vede che questa somma di prodotti diventa precisamente quella che occorre nel teorema succitato di NETTo; noi duremo appunto a ciascun termine il segno +o - secondochè +o sarebbe il segno del corrispondente termine della formola di Neтto. Ora io dico che questa somma è eguale a:

$$
A \cdot\left(a_{1} \ldots a_{m-\lambda} b_{m-\lambda+1} \ldots b_{m}\right)^{i-1}
$$

se $a_{1} \ldots a_{m-\lambda}$ sono le $m-\lambda$ colonne fisse di $A$, e $b_{m-\lambda+1} \ldots b_{m}$ sono le $\lambda$ colonne di $B$ della seconda categoria.

La dimostrazione del teorema la facciamo dipendere dal teorema dato nel paragrafo precedente.

Moltiplichiamo ogni termine della somma (che è sempre un elemento di $D_{m-\lambda+k_{1}}$ moltiplicato per uno di $D_{m-\lambda+k_{2}}$, ecc.) per:

$$
A^{\lambda-k_{3}-1} \cdot A^{\lambda-k_{3}-1} \ldots A^{\lambda-k_{i}-1} \text {. }
$$

Mantenendo le stesse notazioni del paragrafo precedente, ogni termine della somma diventa il prodotto di un minore di ordine $\lambda-k_{1}$ di $D^{(\lambda)}$ moltiplicato per un minore di ordine $\lambda-k_{2}$, e così di seguito. Se in luogo di questi minori si sostituiscono i loro complementi in $D^{(2)}$, si ha una somma di prodotti che non è altro che lo sviluppo secondo la regola di LapLace del determinante $D^{(\lambda)}$. La somma considerata avanti, moltiplicata per quella potenza di $A$, non è altro quindi che ciò che si ottiene facendo lo sviluppo di $D^{(\lambda)}$ secondo la regola di LAPLACE, e poi sostituendo ad ogni minore il suo complemento. Si può ritenere come teorema noto, e del resto potrebbe facilmente dimostrarsi ( $v$. mia Nota citata) che cosi facendo si ha la potenza $i-1^{\text {ma }}$ di $D^{(\lambda)}$; dunque possiamo infine conchiudere che la nostra somma 
moltiplicata per:

è eguale $a$ :

$$
A^{(\lambda-1) i-\left(k_{1}+\ldots+h_{i}\right)}
$$

$$
\left[D^{(\lambda)}\right]^{i-1}
$$

cioè (v. paragrafo precedente) a :

$$
\left[A^{\lambda-1}\left(a_{1} \ldots a_{m-\lambda} b_{m-\lambda+1} \ldots b_{m}\right)\right]^{i-1}
$$

dunque si ha infine che la somma da noi formata è eguale a:

$$
A \cdot\left(a_{1} \ldots a_{m-2} b_{m-\lambda+3} \ldots b_{m}\right)^{i-1},
$$

giusta il teorema enunciato.

Possiamo similmente enunciare un teorema che è analogo a questo e che è a sua volta l'estensione di quell'altro cui ho accennato, analogo al teorema di NetTo:

7. Formiamo la stessa somma precedente, wa sostituendo ad ogni fattore di un termine (fattore che è un determinante con $m-\lambda$ colonne fisse di $A$, con $k_{1}$ altre colonne $d i A$, e con $\left.\lambda-k_{1} d i B\right)$, l'altro determinante, che potrebbe dirsi complementare, cioè formato colle stesse $m-\lambda$ colonne di $A$, poi colle restanti $\lambda-k_{1}$ colonne $d i A$, e colle restanti $k_{1}$ colonne $d i B$. Si ha allora una somma di prodotti di i fattori, che è eguale a:

$$
A^{i-1}\left(a_{1} \ldots a_{n-\lambda} b_{n-\lambda+1} \ldots b_{m}\right) \text {. }
$$

La dimostrazione si fa come quella di avanti, moltiplicando ogni termine per:

$$
A^{k_{1}-1} A^{k_{2}-1} \ldots A^{k_{i}-1} \text {, }
$$

e osservando che allora si ha esattamente lo sviluppo per minori secondo la regola di LAPLACE del determinante $D^{(\lambda)}$, e quindi si ha:

$$
A^{\lambda-1}\left(a_{1} \ldots a_{m-\lambda} b_{m-\lambda+1} \ldots b^{m}\right),
$$

e sopprimendo al primo e secondo membro una opportuna potenza di $A$, resta la formola annunciata.

Pavia, febbraio del 1896. 